\title{
ON THE CONSISTENCY OF QUANTUM FIELD THEORY ${ }^{1}$
}

\author{
BY REESE T. PROSSER
}

Communicated by Ralph Phillips, March 7, 1963

Since its inception some thirty years ago the theory of quantum fields has come to be recognized as a fundamental component in any comprehensive description of the microcosmic physical world. The outstanding successes of the theory in this role indicate that its central features will almost surely persist in some form in whatever revisions of our view of the world are required by the discovery of new evidence. Yet in spite of its successes, this theory has been marred by the presence of internal difficulties, and the stubborn persistence of these difficulties against the best efforts of two generations has led to the wide-spread belief that the theory must somehow be founded on incompatible assumptions.

The question of the consistency of quantum field theory can be restated in purely mathematical terms, and in this form it becomes susceptible to a rigorous mathematical analysis. Our purpose here is to describe briefly the form this question takes and to present a program for resolving it which seems to us to offer a chance of success.

We begin by recalling that quantum field theory assumes that the physical world is made up of elementary particles of various types, and that each type of particle is described by a quantum field $\phi$ satisfying at least the following conditions [3].

(1) Quantum condition. $\phi$ is a tempered distribution defined on the Lorentz space-time manifold with values in a ring of (unbounded) operators acting on a fixed Hilbert space $\mathcal{K}$.

(2) Covariance condition. $\phi$ transforms covariantly according to a given (physically admissible) unitary representation $U$ of the underlying Lorentz group.

(3) Local commutativity. $[\phi(f), \phi(g)]_{ \pm}=0$ whenever the test functions $f$ and $g$ have simultaneous supports. ${ }^{2}$

(4) Existence of a vacuum. There exists a vector $\omega$ in $\mathfrak{K}$, invariant under $U$, which lies in the domain of all polynomial combinations of the field operators.

Examples of fields satisfying these conditions are known; they are the so-called free fields. There is essentially one irreducible free field

\footnotetext{
1 Operated with support from the U. S. Army, Navy, and Air Force.

2 The choice of sign here is determined by $U$, via the well-known connection between spin and statistics.
} 
for each irreducible physically admissible representation of the Lorentz group [2]. These fields cannot be used, however, to describe interactions among particles, and apart from obvious combinations, no other quantum fields are known.

The thing that characterizes a particular quantum field $\phi$, of course, is its behavior under $U$. In practice this behavior is determined by specifying the connection between $\phi$ and $U$, either directly, by expressing the generators of $U$ (i.e., the linear and angular momentum tensors) in terms of $\phi$, or indirectly, by prescribing conditions on $\phi$ via a system of field equations or an action principle. The actual prescription is severely limited by considerations of invariance and simplicity. In addition we shall require that the resulting fields admit a scattering operator, in the sense described below. Apparently only these fields are of any physical interest; these we shall call physical fields. We shall say that a physical field is trivial if the associated scattering operator reduces to the identity, since such fields are physically indistinguishable from free fields.

The consistency problem may now be restated as follows:

Do there exist any nontrivial physical quantum fields?

We maintain that the answer is affirmative, and we present here a program for establishing the existence of these fields. This program consists of implementing the construction of these fields sketched by F. J. Dyson [1] with a careful analysis of the underlying mathematical difficulties.

We begin by fixing a time frame and prescribing in this frame a complete set of free fields $\left\{\phi_{i}\right\}$, one for each type of particle in the system under study. These fields will play the role of the incoming fields. In terms of the incoming fields we form the complete Hamiltonian $H$ of the system as a sum of a free term $H_{0}$, governing the behavior of the incoming fields, and an interaction term $H_{1}$ determining the behavior of the coupled system. For most systems of physical interest it is well known that the free term is well-defined and essentially self-adjoint on the state space associated with the incoming fields. The interaction term, on the other hand, usually involves products of the incoming fields which are $a$ priori undefined and indeed undefinable. In order to circumvent this difficulty we shall provisionally replace each of the free fields $\phi_{i}$ appearing in the interaction term by a modified field $\phi_{i}(\rho)$ defined by the relation

$$
\phi_{i}(\rho)=\rho * \phi_{i}
$$

where $*$ denotes a four-dimensional convolution, and $\rho$ a suitably chosen scalar factor of the form $\rho(x)=\rho(x) \delta(t)$. Here $\rho(x)$ approxi- 
mates $\delta(x)$ and is sufficiently smooth to ensure that all relevant products are well-defined, but is otherwise quite arbitrary. The essential dynamical properties of the total Hamiltonian are not affected by this modification, so long as we do not change the time frame.

Our first task is to establish

Proposition 1. $H$ is well-defined and essentially self-adjoint on the free state space.

The interaction term $H_{1}$ will contain a number of parameters, including mass, charge, and scale corrections, which are in principle unobservable and hence remain at our disposal. By suitably adjusting these parameters, we proceed to modify the total Hamiltonian so that its spectrum includes that of the free Hamiltonian. Thus we establish

Proposition 2. The spectrum of $H$ includes the spectrum of $H_{0}$.

Next we form the unitary operators $\exp \left(i H_{0} t\right)$ and $\exp (i H t)$, and put $W(t)=\exp (-i H t) \exp \left(+i H_{0} t\right)$ and $W\left(t, t^{\prime}\right)=W^{-1}(t) W\left(t^{\prime}\right)$. In terms of these operators we formulate

Proposition 3. The weak limits $W(t, \pm \infty)=\lim _{t^{\prime} \rightarrow \pm \infty} W\left(t, t^{\prime}\right)$ exist, and satisfy the relation $H W(0, \pm \infty)=W(0, \pm \infty) H_{0}$.

This limit is a bounded operator on $\mathcal{H}$, but it need not be unitary, and it is conceivable that it be identically zero. In order to exclude this possibility, we formulate

Proposition 4. If $f$ is any state $\neq 0$, then $W(t, \pm \infty) f \neq 0$.

Now if we resolve $W(t, \pm \infty)$ according to the canonical polar decomposition as a product $U(t, \pm \infty) T$, with $U(t, \pm \infty)$ a partial isometry and $T$ positive, then it is clear from the propositions above that $U(t, \pm \infty)$ must be semi-unitary, and that $T$ is independent of $t$, commutes with $H_{0}$, and admits an inverse $T^{-1}$ (perhaps unbounded), with $T^{-1} \geqq I$. If we put $W_{r}\left(t, t^{\prime}\right)=T^{-1} W\left(t, t^{\prime}\right) T^{-1}$, then $W_{r}\left(t, t^{\prime}\right)$ is well defined on the domain of $T^{-1}$ and satisfies the following weak limit relations.

$$
\begin{aligned}
\lim _{t^{\prime} \rightarrow \pm \infty} W_{r}\left(t, t^{\prime}\right) & =T^{-1} U(t, \pm \infty), \\
\lim _{t \rightarrow \pm \infty} \lim _{t^{\prime} \rightarrow \pm \infty} W_{r}\left(t, t^{\prime}\right) & =I, \\
\lim _{t \rightarrow+\infty} \lim _{t^{\prime} \rightarrow-\infty} W_{r}\left(t, t^{\prime}\right) & =U(0,+\infty)^{-1} U(0,-\infty)=S_{r} .
\end{aligned}
$$

In particular, the renormalized scattering operator $S_{r}$ defined in this way 
is unitary whenever the ranges of $W(0,-\infty)$ and $W(0,+\infty)$ coincide.

Now we turn to the coupled fields. We define the Heisenberg fields $\phi_{h}$ in terms of the incoming free fields $\phi_{f}$ via the relation

$$
\phi_{h}(t)=U(t,-\infty) \phi_{f}(t) U(t,-\infty)^{-1} .
$$

The Heisenberg fields are then unitarily equivalent with the free fields, and their time dependence is governed by the free Hamiltonian $H_{0}$ (which is related to the Heisenberg fields in the same way that the total Hamiltonian $H$ is related to the free fields) by the relation

$$
\phi_{h}(t)=e^{i H_{0} t} \phi_{h}(0) e^{-i H_{0} t} .
$$

As $t \rightarrow-\infty$, however, the Heisenberg fields do not necessarily reduce to the free fields. In fact, if we resolve the free fields into positive and negative frequencies, $\phi_{f}=\phi_{f}^{+}+\phi_{f}^{-}$, and define

$$
\phi_{h}^{ \pm}(t)=U(t,-\infty) \phi_{f}^{ \pm}(t) U(t,-\infty)^{-1},
$$

then we find that as $t \rightarrow-\infty$,

$$
\begin{aligned}
\phi_{h}^{+}(t) & \sim T^{-1} \dot{\phi}_{f}^{+}(t) T, \\
\overline{\phi_{h}}(t) & \sim T \overline{\phi_{f}}(t) T^{-1} .
\end{aligned}
$$

This suggests that we define the renormalized fields according to

$$
\begin{aligned}
\phi_{r}^{+}(t) & =T \phi_{h}^{+}(t) T^{-1}, \\
\overline{\phi_{r}}(t) & =T^{-1} \overline{\phi_{h}}(t) T, \\
\phi_{r}(t) & =\phi_{r}^{+}(t)+\overline{\phi_{r}}(t)
\end{aligned}
$$

and establish

Proposition 5. The renormalized fields are weakly asymptotic to the incoming fields as $t \rightarrow-\infty$.

The functional dependence of the renormalized scattering operator $S_{r}$ upon the renormalized fields is exactly the same as that of the unrenormalized scattering operator $S=T S_{r} T=W(0,+\infty)^{-1} W(0,-\infty)$ upon the Heisenberg fields; namely, via the Dyson expansion. Thus the usual rules of computation remain valid, though in some cases the perturbation series may not converge. The functional dependence of the free and total Hamiltonians upon the renormalized fields is less straightforward and apparently has no simple form.

All of the preceding considerations are based on the presence of the form factors $\rho$ in the interaction Hamiltonian. It remains to see what 
happens when these form factors are removed. More precisely, we let each of the form factors $\rho(x)$ tend to the point factor $\delta(x)$ in an appropriate sense of convergence and investigate the behavior of the various expressions we have developed in this limit. The key results can be formulated as follows:

Proposition 6. As $\rho \rightarrow \delta$ the renormalized scattering operator $S_{r}$ admits a strong limit, independent of the choice of $\rho$.

Proposition 7. As $\rho \rightarrow \delta$ the renormalized fields $\phi_{r}$ admit a strong limit as four-dimensional operator-valued distributions, independent of the choice of $\rho$.

PROPOSITION 8. The limiting renormalized fields satisfy the quantum field conditions (1)-(4).

PROPOSITION 9. The limiting renormalized fields are weakly asymptotic to the free fields $\phi_{\text {in }}$ as $t \rightarrow-\infty$ and $\phi_{\text {out }}$ as $t \rightarrow+\infty$, and these fields are related by the formula $\phi_{\text {out }}=S_{r} \phi_{\mathrm{in}} S_{r}^{-1}$.

Neither $W\left(t, t^{\prime}\right)$ and $W_{r}\left(t, t^{\prime}\right)$, nor $W(t, \pm \infty)$ and $U(t, \pm \infty)$, nor the Heisenberg fields, nor the instantaneous renormalized fields will in general admit limits as $\rho \rightarrow \delta$. Only the renormalized scattering operator and space-time averages of the renormalized fields admit these limits. Moreover, the limiting fields are not unitarily equivalent with the free fields, and the so-called interaction representations of these fields do not exist. This fact accounts for the difficulties in dealing with these fields and explains the necessity for introducing form factors in the Hamiltonians.

Proposition 9 completes our program for the construction of the required quantum fields.

We have succeeded in carrying out the first part of this program for those systems for which the dependence of the total Hamiltonian upon the Boson fields is at most quadratic. For such systems we have rigorously established the validity of Propositions (1)-(5) for all values of the coupling parameter. These systems include the photonelectron system, the pion-nucleon system, and the four-Fermion system, but exclude the pion-pion system and its analogues. A recent result of Galindo [4] indicates that the situation is quite different for multiple Boson interactions, and that our results cannot be expected to apply.

We have also established the validity of Propositions (6)-(9) for certain special cases, including ${ }^{3}(1)$ a Boson field with external scalar

${ }^{3}$ In each case the role of the Lorentz group is taken over by whatever symmetry group is appropriate for the system. 
source, (2) a Fermion field with external scalar source, (3) a Fermion field in the Lee model, and (4) a restricted form of the photon-electron system, with no positron states. The extension of these results to the complete photon-electron system is now under study.

In the case of the Lee model, we found that the limiting renormalized Fermion field produced by our program is identical with the incoming free field, and hence is trivial. This can be avoided only by requiring that the (unrenormalized) coupling constant be complex. At first sight this procedure seems to destroy the validity of the whole theory, since then the total Hamiltonian is apparently no longer selfadjoint. But a closer investigation reveals that actually it is the Heisenberg fields which are not self-adjoint; the total Hamiltonian constructed from these fields is self-adjoint, and in fact is equal to the free Hamiltonian constructed from the incoming free fields. Moreover, all of our results depend analytically upon the coupling constant, and hence remain valid for complex as well as real values of this parameter, provided we generalize suitably the notions of selfadjoint and unitary operators. Thus for complex coupling constants it can happen that the Heisenberg fields are not self-adjoint and yet the renormalized fields are, that the wave operators $U(0, \pm \infty)$ are not unitary, and yet the scattering operator is. In this case the final results are perfectly valid; only the original formulation, in terms of Heisenberg fields, is at fault. At any rate this argument is certainly the key to the puzzle of the Lee model, and probably also plays an essential role in the complete photon-electron system.

\section{REFERENCES}

1. F.J. Dyson, The S-matrix in quantum electrodynamics, Phys. Rev. (2) 75 (1949), 1736-1755.

2. I. E. Segal, Foundations of the theory of dynamical systems of infinitely many degrees of freedom, Mat.-Fys. Skr. Dansk Vid. Selsk. 31 (1959), 1-39.

3. A. S. Wightman, Les problèmes mathématiques de la thêrie quantique des champs, Colloques Internationaux du Centre National de la Recherche Scientifique LXXV, Centre National de la Recherche Scientifique, Paris, 1959, pp. 1-38.

4. A. Galindo, On a class of perturbations in quantum field theory, Proc. Nat. Acad. Sci. U.S.A. 48 (1962), 1128-1131.

LINCOLN LABORATORY 